\title{
Effects of autophagy and endocytosis on the activity of matrix metalloproteinase- 2 in human renal proximal tubular cells under hypoxia
}

\author{
WENMIN YU ${ }^{1,2}$, ZHI WANG ${ }^{2}$, YIPING LI ${ }^{2}$, LEI LIU ${ }^{2}$, JING LIU ${ }^{2}$, FENGGAN DING $^{2}$, \\ XIAOYI ZHANG ${ }^{2}$, ZHENGYUAN CHENG $^{2}$ and PINGSHENG CHEN ${ }^{2}$
}

\begin{abstract}
${ }^{1}$ The School of Basic Medical Science, Jiujiang University/Jiujiang Key Laboratory of Translational Medicine, Jiujiang, Jiangxi 332000; ${ }^{2}$ Department of Pathology and Pathophysiology, Medical School of Southeast University, Nanjing, Jiangsu 210009, P.R. China
\end{abstract}

Received February 4, 2016; Accepted January 30, 2017

DOI: $10.3892 / \mathrm{mmr} .2017 .6358$

\begin{abstract}
Tubulointerstitial fibrosis is characterized by tubular atrophy with basement membrane thickening and accumulation of interstitial extracellular matrix (ECM). A decrease in the activity of matrix metalloproteinase-2 (MMP-2) may promote this process. Although proximal tubular cells are sensitive to oxygen deprivation, whether cellular autophagy or endocytosis induced by hypoxia can alter the activity of MMP-2 remains to be elucidated. The aim of the present study was to investigate whether autophagy and endocytosis induced by hypoxia can have an effect on the activity of MMP-2 in HK-2 cells. The investigations involved exposing the HK-2 cell line to an autophagy inhibitor, 3-MA, or an endocytotic inhibitor, filipin. The mRNA expression of MMP-2 was elevated in the hypoxic milieu. Furthermore, it was found that filipin increased the activity of MMP-2 under hypoxia. These results suggested that autophagy and endocytosis were potential mediators for the altered expression of MMP-2, and endocytosis was a potential target for regulating the activity of MMP-2. These data suggested that hypoxia may be an important pro-fibrogenic stimulus, which acts in part via endocytosis.
\end{abstract}

Correspondence to: Professor Pingsheng Chen, Department of Pathology and Pathophysiology, Medical School of Southeast University, 87 Ding Jiaqiao Road, Nanjing, Jiangsu 210009, P.R. China

E-mail: chenpsh@sina.com

Abbreviations: ECM, extracellular matrix; MMP-2, matrix metalloproteinase-2; MT1-MMP, membrane-type 1 matrix metalloproteinase; CKD, chronic kidney disease; Col-IV, collagen-IV; ELISA, enzyme-linked immunosorbent assay

Key words: proximal tubular cells, hypoxia, autophagy, endocytosis, matrix metalloproteinase-2

\section{Introduction}

Chronic kidney disease (CKD) is increasingly recognized as a worldwide public health issue (1). Chronic hypoxia often occurs in the kidney tissues of several patients with CKD. Tubulointerstitial fibrosis is a pathological characteristic of the development of almost all CKDs (2). Tubulointerstitial fibrosis occurs and leads to increased thickness of the tubular basement membrane (TBM) and accumulation of interstitial ECM (3-6). Matrix metalloproteinase-2 (MMP-2) is involved in the breakdown of ECM, degrading type IV collagen (Coll-IV), a major component of the basement membrane (7-11). The activity of MMP-2 in kidney tissues of patients with CKD is decreased, leading to progressive renal dysfunction and organ failure (12), although the mechanism remains to be fully elucidated.

As is already known, hypoxia alters the expression levels of the components of the tissue inhibitors of metalloproteinases (TIMP) in human renal tubular endothelia, interstitial fibroblasts and microvascular endothelial cells (13). In vitro studies have found that the activity of MMP-2 is decreased in proximal tubular cells under hypoxic conditions (14). Our previous study demonstrated altered expression and activity of MMP-2 in hepatic stellate cells under hypoxic conditions (15), however, the mechanism remains to be fully elucidated.

Previous studies have shown that autophagy also has a close complex link with hypoxia (16). Extensive data suggests that autophagy has a dual effect in hypoxia-induced cell injury (17). The adaptation to hypoxia at the cellular level is regulated by a dual mechanism; hypoxia leads to augmentation in the efficiency of energy-producing pathways, and decreases energy consuming processes, including the activity of Na, K-ATPase (18). As a cellular adaptive response, hypoxia decreases the activity of Na, K-ATPase by triggering the endocytosis of its $\alpha 1$ subunit in the alveolar epithelia (19). These studies indicated that hypoxia induces autophagy and endocytosis. Another study showed that autophagy and endocytosis lead to reshaping of the cell membrane (20). Membrane-type 1 MMP (MT1-MMP) is a zinc-dependent proteinase found in cholesterol-rich lipid rafts on the plasma membrane. MT1-MMP activates proMMP-2 (21), and the 
cleavage of proMMP-2 to an active form in a ternary complex with TIMP $(22,23)$. Therefore, the present study hypothesized that autophagy and endocytosis triggered by hypoxia can lead to alteration of the plasma membrane, which can alter the expression of molecules regulating the activation of MMP-2, and eventually resulting in a decrease in the activity of MMP-2.

The present study investigated the association between autophagy and endocytosis and the activity of MMP-2 in human proximal tubular cells under hypoxia in vitro. As proximal tubular cells are key in the development of renal fibrosis (24), the results of the present study may provide useful clues to understand the mechanism by which hypoxia alters the activity of MMP-2 in HK-2 cells.

\section{Materials and methods}

Cell culture and hypoxic treatment. The HK-2 human renal proximal tubular epithelial cell line was purchased from the Cell Bank of Type Culture Collection of Chinese Academy of Sciences (Shanghai, China). The HK-2 cells were cultured in high-glucose DMEM supplemented with $10 \%$ fetal bovine serum (FBS), $100 \mathrm{U} / \mathrm{ml}$ penicillin and $100 \mu \mathrm{g} / \mathrm{ml}$ streptomycin (Thermo Fisher Scientific, Inc., Waltham, MA, USA) in humidified air containing $5 \% \mathrm{CO}_{2}$ at $37^{\circ} \mathrm{C}$. At the second passage of the cell culture, the viability of the cells was optimal for experiments, which was the phase identified as the logarithmic growth phase. Cells in the logarithmic growth phase were used in all experiments.

The cells were seeded on 6-well plates at a density of $5 \times 10^{5}$ cells/well and cultured for $24 \mathrm{~h}$. To mimic hypoxic conditions, the cells in the culture medium were then subjected to low-oxygen conditions. The oxygen concentrations were maintained at 1-3\% using a three gas incubator (Thermo Fisher Scientific, Inc.), which was held under a positive pressure in an atmosphere of 94-92\% $\mathrm{N}_{2} / 5 \% \mathrm{CO}_{2} / 1-3 \% \quad \mathrm{O}_{2}$ for $24 \mathrm{~h}$. For the hypoxic experiments, there were four groups of triplicate wells, comprising a control group, hypoxic group, hypoxic+3-methyladenine (3-MA) group (5 mM 3-MA; Sigma-Aldrich; Merck KGaA, Darmstadt, Germany) and a hypoxic+filipin group (filipin $2.5 \mu \mathrm{g} / \mathrm{ml}$; Sigma-Aldrich; Merck KGaA) group for $24 \mathrm{~h}$ at $37^{\circ} \mathrm{C}$. The control cells were cultured under normoxic conditions.

Western blot analysis. The cells were plated at a density of $5 \times 10^{5}$ cells/well on 6 -well plates and cultured in DMEM supplemented with $10 \%$ FBS for $24 \mathrm{~h}$. Following treatment under hypoxia for $24 \mathrm{~h}$, the cells were washed three times with ice-cold 1X PBS and lysed with immunoprecipitation assay buffer containing protease inhibitors. Following incubation on ice for $15 \mathrm{~min}$ to ensure complete lysis, the cell lysates were centrifuged at $14,000 \mathrm{~g}$ for $10 \mathrm{~min}$ at $4^{\circ} \mathrm{C}$. The protein concentrations of the lysates were examined using a Bradford protein assay kit. The cell lysates were boiled and $40 \mu \mathrm{g}$ of proteins were separated by $10 \%$ sodium dodecyl sulfate-polyacrylamide gel electrophoresis (SDS-PAGE), and transferred onto a polyvinylidene difluoride membrane via semi-dry transfer (Bio-Rad Laboratories Inc., Hercules, CA, USA). The membranes were washed in Tris-buffered saline containing $0.1 \%$ Tween-20 (TBST), blocked with 5\% non-fat milk in TBST for $1 \mathrm{~h}$ at room temperature, and incubated with primary rabbit monoclonal antibody against LC3B (ab48394; Abcam, Cambridge, UK; 1:1,000), anti-MT1-MMP (ab51074; Abcam; 1:1,000), anti-caveolin-1 (16447-1-AP; Protech Technology, Inc., China; 1:1,000) or glyceraldehyde-3-phosphate dehydrogenase (GAPDH; 10494-1-AP; Protech Technology, Inc.; $1: 5,000)$ overnight at $4^{\circ} \mathrm{C}$. The membranes were washed three times in TBST, followed by incubation with the appropriate horseradish peroxidase (HRP)-linked secondary anti-rabbit antibodies (SA00001-1; Proteintech, Wuhan, China; 1:5,000) for $1 \mathrm{~h}$ at room temperature. The specific proteins on the blots were developed using enhanced chemiluminescence (ECL; Vazyme Biotech Co., Ltd., Nanjing, China) and visualized as bands on CL-XPosure film (Thermo Fisher Scientific, Inc.). The optical densities of the bands were measured on the GS710 Densitometer and analyzed using Quantity One image analysis software version 4.6 (Bio-Rad Laboratories, Inc.).

Reverse transcription-quantitative polymerase chain reaction (RT-qPCR) analysis. Total RNA was extracted from the HK-2 cells using the RNeasy Mini kit (Vazyme Biotech Co., Ltd.), and DNase digestion was executed using an RNase-free DNase set (Vazyme Biotech, Co., Ltd.). RT-qPCR was performed to determine the expression of human MMP-2 using SYBR Green PCR Master mix (Vazyme Biotech Co., Ltd.) using the StepOnePlus $^{\text {TM }}$ Real-Time PCR Detection system (Step One Plus 2.1 software) with universal thermal cycling parameters, Reverse transcription was performed in a reaction system containing $5 \mu \mathrm{g}$ RNA, reverse transcriptase, RNase inhibitor and random primers. The obtained cDNAs were used to run qPCR with pairs of primers. The thermal cycling conditions were as follows: $10 \mathrm{sec}$ at $95^{\circ} \mathrm{C}$ followed by the amplification reaction consisting of 40 cycles of denaturation for $10 \mathrm{sec}$ at $95^{\circ} \mathrm{C}$ and annealing for $30 \mathrm{sec}$ at $60^{\circ} \mathrm{C}$. The resulting data were analyzed with the comparative $\mathrm{Ct}$ method for relative gene expression quantification against housekeeping gene, GAPDH. The following primers were used: MMP-2 forward 5'-GAG AACCAAAGTCTGAAGAG-3' and reverse 5'-GGAGTG AGAATGCTGATTAG-3'; GAPDH forward 5'-GGAAGG TGAAGGTCGGAGTCA-3' and reverse 5'-GCAACAATA TCCACTTTACCAG-3'. The gene expression level of GAPDH served as the control of target genes for reaction efficiency. The $2^{-\Delta \Delta \mathrm{Cq}}$ method (25) was used to determine the relative quantities of products.

Enzyme-linked immunosorbent assay (ELISA) to quantify MMP-2 and Collagen-IV (Col-IV) in culture media. ELISA kits were used to detect the protein contents of MMP-2 and Col-IV in culture media, according to the manufacturer's protocol (E-11117 and E-13368 respectively; Shanghai Hengyuan Biological Technology, Co., Ltd., Shanghai, China). Purified MMP-2 or Col-IV antibody was used to coat the microtiter plate wells, and the culture supernatants were then mixed into the wells and incubated at $37^{\circ} \mathrm{C}$ for $2 \mathrm{~h}$. Following washing of the plates with PBS, another MMP-2 antibody or Col-IV antibody labeled with HRP was added to the plates at $37^{\circ} \mathrm{C}$ for $2 \mathrm{~h}$. The plates were then washed thoroughly and 3,3',5,5'-tetramethylbenzidine substrate solution was added. The reaction was terminated by the addition of a sulfuric acid solution, and color was measured by spectrophotometry at a wavelength $450 \mathrm{~nm}$. The protein contents of MMP-2 or Col-IV 
in the samples were then calculated according to the standard curve using Origin version 8.0 (OriginLab, Northampton, MA, USA).

Detection of the activity of MMP-2 using zymography. The activity of MMP-2 was determined via gelatin zymography using an MMP Zymography Assay kit (Applygen Technologies, Inc., Beijing, China) according to the manufacturer's protocol. Following MMP-2 separation using SDS-PAGE, the SDS was extracted from the gels using Triton X-100, and then incubated for $48 \mathrm{~h}$ at $37^{\circ} \mathrm{C}$. The gels were stained with coomassie brilliant blue G250 and decolorized. The clear band against a blue background represented the activity of MMP-2 and was measured using a gel image system (Image Master 1D analysis software; GE Healthcare Life Sciences, Shanghai, China) and recorded as the total area (area of clear band $\mathrm{x}$ mean area) (26).

Statistical analysis. Statistical analyses were performed using the SPSS 13.0 statistical software package (SPSS, Inc., Chicago, IL, USA). The data are expressed as the mean \pm standard deviation. Differences between groups were analyzed using Student's t-test. $\mathrm{P}<0.05$ was considered to indicate a statistically significant difference.

\section{Results}

3-MA and filipin inhibit hypoxia-induced autophagy and endocytosis in HK-2 cells, respectively. To examine the inhibition of 3-MA and filipin in the HK-2 cells subjected to hypoxia, the expression of LC3 and Cavelin-1 were detected $\mathrm{n}$ in HK-2 cells under hypoxia using western blot analysis. The results of the western blot analysis of LC3 confirmed that there was increased transformation from LC3-I to LC3-II in the hypoxia-treated HK-2 cells, compared with the cells under normoxic conditions, which demonstrated that hypoxia induced autophagy. However, in the hypoxic cells treated with 3-MA, the transformation from LC3-I to LC3-II was significantly reduced. These data suggested that hypoxia induced autophagy in HK-2 cells, which was inhibited by 3-MA (Fig. 1A and B). Simultaneously, it was found that hypoxia upregulated the protein expression of Caveolin-1. When the HK-2 cells were treated with filipin under hypoxia, the expression of Caveolin-1 was inhibited (Fig. 1A and C). These results showed that hypoxia promoted autophagy and Caveolae endocytosis, which were inhibited by 3-MA and filipin in HK-2 cells under hypoxia, respectively.

Inhibition of autophagy and endocytosis alters the activity of MMP-2 in culture media of hypoxia-treated HK-2 cells. The results of the gel zymography of MMP-2 showed two major bands, one band at $62 \mathrm{kDa}$ representing the active form of MMP-2 and another at $72 \mathrm{kDa}$ representing pro-MMP-2. The activity of MMP-2 in the hypoxic group $(0.36 \pm 0.07)$ was found to be lower, compared with that in the normoxic group $(1.00 \pm 0.00)$. When the HK-2 cells were treated with 3-MA, active MMP-2 $(0.25 \pm 0.04)$ in the hypoxic+3-MA group was further decreased, compared with that in the hypoxic group. By contrast, the activity of MMP-2 was significantly elevated in the group treated with filipin and hypoxia $(0.60 \pm 0.08)$, compared with that in the hypoxic group $(\mathrm{P}<0.01$; Fig. $2 \mathrm{~A}$ and $\mathrm{B})$.
Inhibition of autophagy and endocytosis enhances the mRNA expression of MMP-2 in hypoxia-treated $H K$ - 2 cells. To examine the expression of MMP-2 and its correlation with autophagy and endocytosis, the present study investigated the expression of MMP-2 in HK-2 cells using RT-qPCR analysis. Compared with the normoxic group, the mRNA level of MMP-2 was not affected in the cells exposed to hypoxia. However, 3-MA and filipin significantly increased the mRNA levels of MMP-2 in the hypoxic cells $(\mathrm{P}<0.01)$, and 3-MA exhibited more marked induction of the mRNA expression of MMP-2, compared with filipin. The relative expression of MMP-2 was $1.24 \pm 0.16$ in the hypoxic group, $15.53 \pm 2.12$ in the hypoxic $+3 \mathrm{MA}$ group and $6.40 \pm 4.22$ in the hypoxic+filipin group (Fig. 2C).

Inhibition of autophagy and endocytosis alters the protein levels of MMP-2 and Col-IV in the culture media of hypoxia-treated HK-2 cells. The protein expression levels of MMP-2 in cell culture media was determined using ELISA to determine the efficiency of protein secretion by the cells. As shown in Fig. 2D, the protein expression of MMP-2 $(3.71 \pm 0.12 \mu \mathrm{g} / \mathrm{l})$ was significantly reduced in the hypoxia-treated cells, compared with that in the normoxic cells $(4.54 \pm 0.33 \mu \mathrm{g} / \mathrm{l}$; $\mathrm{P}<0.05)$. When the cells were incubated with the inhibitor, 3-MA, under hypoxia for $24 \mathrm{~h}$, the protein expression of MMP-2 (3.67 $\pm 0.20 \mu \mathrm{g} / \mathrm{l})$ was further reduced, compared with the hypoxic group. However, the addition of filipin increased the protein level of MMP-2 in the culture media $(4.44 \pm 0.31 \mu \mathrm{g} / \mathrm{l})$, compared with that in the hypoxic group $(\mathrm{P}<0.01$; Fig. $3 \mathrm{~A})$.

As the protein level of Col-IV in the cell culture media was opposite to the activity of MMP-2, the protein expression of Col-IV was examined using ELISA to assess the enzymatic activity of MMP-2. The results showed that the protein levels of Col-IV in the hypoxic group $(5.33 \pm 0.14 \mu \mathrm{g} / \mathrm{l})$ and hypoxic+3-MA group $(5.46 \pm 0.06 \mu \mathrm{g} / \mathrm{l})$ were increased, compared with that in the normoxic group $(5.02 \pm 0.07 \mu \mathrm{g} / \mathrm{l})$, suggesting decreased activity of MMP-2. The protein level of Col-IV was decreased in the hypoxic+filipin group $(5.18 \pm 0.02 \mu \mathrm{g} / \mathrm{l})$, compared with that in the hypoxic group $(\mathrm{P}<0.05$; Fig. 3B), indicating increased activity of MMP-2 in the presence of filipin.

Inhibition of endocytosis increases the expression of MT1-MMP in hypoxia-treated HK-2 cells. Western blot analysis was used to detect the protein levels of MT1-MMP in the cells. The protein expression of MT1-MMP was normalized with the internal control, GAPDH (Fig. 3C). The results showed that the protein expression of MT1-MMP was significantly higher in the normoxic group, compared with that in the hypoxic group. Compared with the hypoxic group, treatment with 3-MA under hypoxic conditions for $24 \mathrm{~h}$ resulted in a further decrease in the protein level of MT1-MMP, whereas filipin increased the protein level of MT1-MMP in the cells. The fold increases in MT1-MMP protein were $0.54 \pm 0.09$ and $0.80 \pm 0.12$, compared with the normoxic group, respectively (Fig. 3D).

\section{Discussion}

Renal hypoxia is an important factor in the pathophysiology of the acute kidney injury (AKI) to CKD transition (27). The reason for the reduced MMP-2 activity in patients with CKD remains to be fully elucidated. Studies have shown that 
A

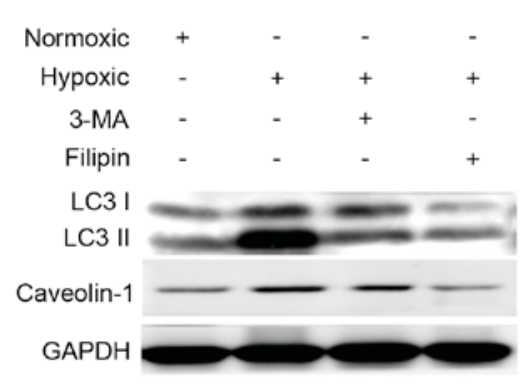

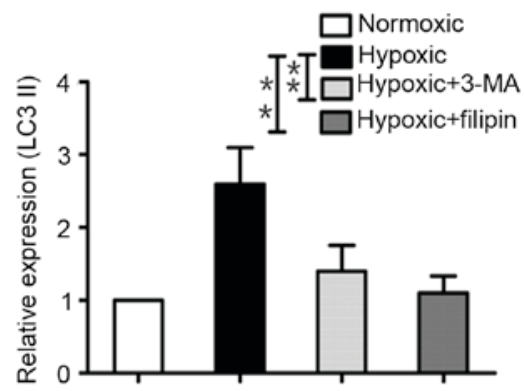

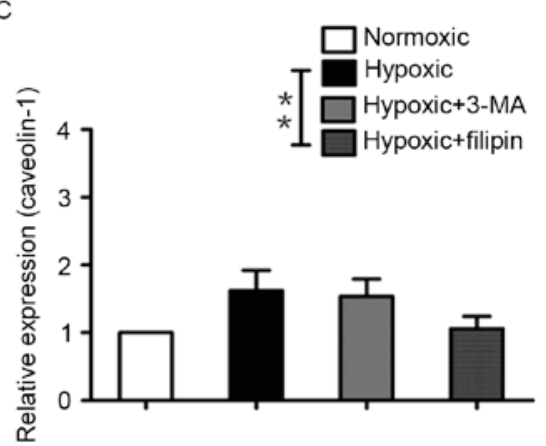

Figure 1.3-MA and filipin inhibit the autophagy and endocytosis induced by hypoxia in HK-2 cells. (A) Western blot analysis of the autophagy protein (LC3B) and endosome proteins (Caveolin-1) in HK-2 cells exposed to hypoxia and 3-MA or filipin for $24 \mathrm{~h}$. (B) Expression of LC3-II relative to that of GAPDH. (C) Expression of Caveolin-1 relative to that of GAPDH. Data are presented as the mean \pm standard deviation, and results were analyzed using Student's t-test (unpaired) ${ }^{* *} \mathrm{P}<0.01$. 3-MA, 3-methyladenine; LC3B, microtubule-associated protein light chain 3.
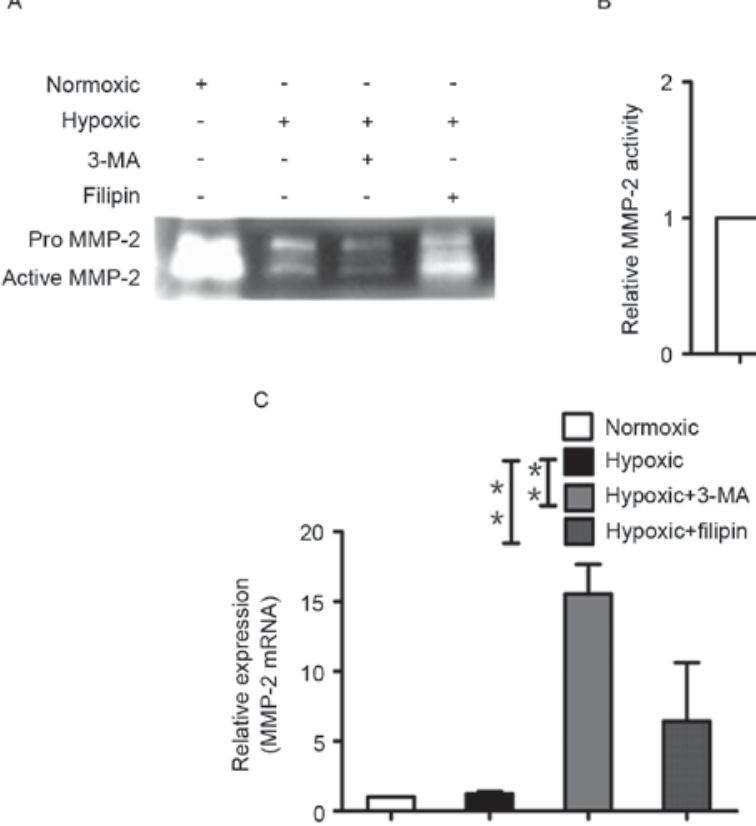

Figure 2. 3-MA and filipin alter the activity of MMP-2 in culture media of HK-2 cells under hypoxia. (A) MMP-2 activity was evaluated using gelatin-substrate gel zymography in culture media of hypoxia-treated HK-2 cells. (B) Gels were scanned and quantified using densitometry, and relative MMP-2 activity was calculated as a percentage of the relevant control values (assigned as 1) in arbitrary densitometry units. Data are presented as the mean of three separate experiments. (C) Relative mRNA levels were calculated as a percentage of the relevant control values. Results were analyzed using Student's t-test (unpaired) ${ }^{*} \mathrm{P}<0.05 ;{ }^{* *} \mathrm{P}<0.01$. MMP-2, matrix metalloproteinase- 2 .

renal proximal tubular cells may be the primary target of a hypoxic insult $(1,28)$. Hypoxia induces autophagy and endocytosis (17-19). However, whether hypoxic-induced autophagy and Caveolae-mediated endocytosis can affect the activity

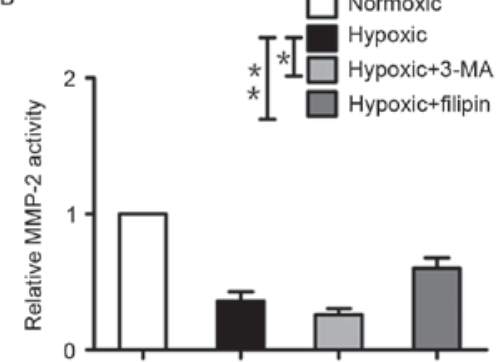


A

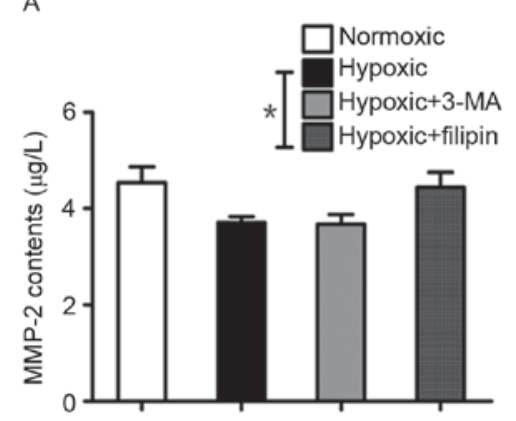

C

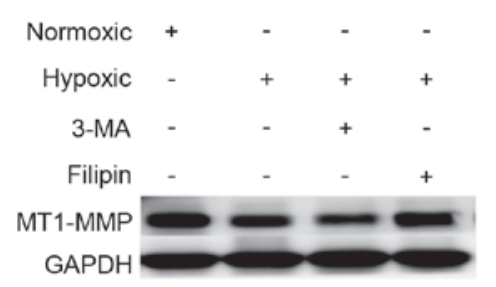

B

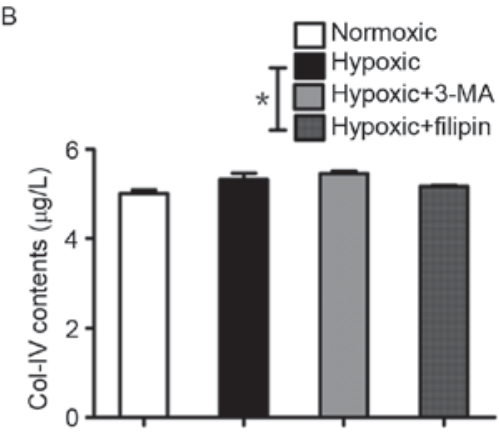

D

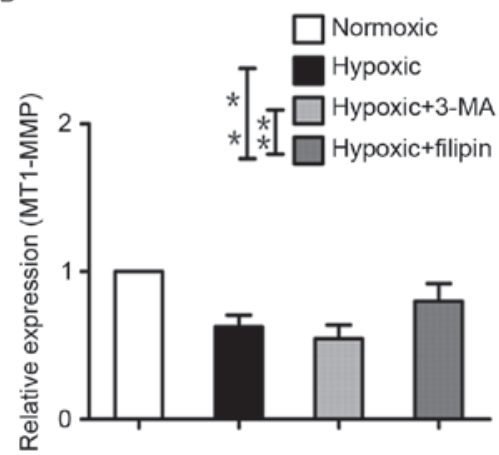

Figure 3. Protein expression of MMP-2 and Col-IV in cell culture supernatants, and of MT1-MMP in hypoxia-treated HK-2 cells. (A) Protein expression of MMP-2 in cell culture supernatants was detected using ELISA. (B) Protein expression of Col-IV in cell culture supernatants was detected using ELISA. (C) Western blot analysis of the protein levels of MT1-MMP in HK-2 cells exposed to hypoxia and 3-MA or filipin for 24 h. (D) Expression of MT1-MMP relative to that of GAPDH. Data are presented as the mean \pm standard deviation, and results were analyzed using Student's t-test (unpaired) ${ }^{*} \mathrm{P}<0.05 ;{ }^{* *} \mathrm{P}<0.01$. MMP-2, matrix metalloproteinase-2; Col-IV, collagen-IV; MT1, membrane-type 1; ELISA, enzyme-linked immunosorbent assay.

and decreased autophagy and endocytosis were observed in the cells following exposure to hypoxia for $24 \mathrm{~h}$. These data suggested that 3-MA and filipin were effective inhibitors for autophagy and Caveolae-mediated endocytosis in hypoxia, respectively.

In the present study, the specific inhibitors for autophagy and Caveolae-mediated endocytosis altered the expression and activity of MMP-2 in hypoxia-treated HK-2 cells. 3-MA and filipin significantly increased the mRNA levels of MMP-2 in hypoxia-treated cells. In particular, the inhibition of Caveolae-mediated endocytosis by filipin increased the activity of MMP-2 in culture media under hypoxia. Although the inhibition of autophagy considerably upregulated the expression of MMP-2 at the mRNA level, 3-MA decreased the activity of MMP-2 in culture media under hypoxia. In a report by Orphanides et al (14), data suggested that regulation of the activity of MMP-2 was via a post-transcriptional mechanism, and the results of the present study appeared to confirm this, with the ELISA results for the protein expression levels of Col-IV and MMP-2 confirming these observations.

A previous study showed that Caveolin-1 inhibits the activity of MT1-MMP, promoting the internalization of MT1-MMP from the cell surface and reducing the activation of proMMP-2 (21). Another study demonstrated that MT1-MMP is crucial for the activation of proMMP-2 (22). In the present study, the protein level of MT1-MMP was increased, and the activity of MMP-2 was augmented. These findings are consistent with these previous reports. The expression of MT1-MMP induced the activation of proMMP-2, however, the association requires further elucidation. Previous reports have shown that Caveolin-1 inhibits the activity of MMP-2 in heart tissue (29) and filipin decreases Caveolae-mediated endocytosis (30). In the present study, when filipin inhibited the expression of Caveolin-1 under hypoxia, the levels of Caveolin-1 significantly increased the activity of MMP-2, and this result is similar to results of previous studies $(29,30)$.

The activity of MMP-2 is key in preventing the thickness of the TBM and the accumulation of interstitial ECM in patients with CKD (3-6). The present study demonstrated the association of hypoxia-induced autophagy and endocytosis with the activity of MMP-2, respectively, providing novel insight into the mechanisms underlying the interaction between the activity of MMP-2 and hypoxia. Furthermore, these results provide clues regarding the mechanism underlying progressive fibrosis in patients with CKD. It was hypothesized that the activity of MMP-2 increases in culture media following the inhibition of autophagy and endocytosis as molecules regulating the activation of MMP-2 may not be affected, however, only the inhibition of endocytosis increased the activity of MMP-2. It is likely that 3-MA may affect other signaling molecules responsible for regulating the mRNA level and activity of MMP-2 under hypoxic conditions. Further investigation in the in vivo model is warranted.

In conclusion, the present study provided novel findings suggesting that the inhibition of Caveolae-mediated endocytosis may increase the activity of MMP-2 in the kidney tissues of patients with CKD, and may be potentially used to inhibit renal fibrogenesis, prevent TBM thickness and prevent the 
accumulation of ECM in renal diseases. Simultaneously, the results of the present study may provide novel insight into the mechanism underlying progressive fibrosis.

\section{Acknowledgements}

The authors would like to thank Professor Wandong Zhang at the National Research Council of Canada (Ottawa, ON, Canada) for critically reading and editing the manuscript. The present study was financially supported by the National Nature Science Foundation of the People's Republic of China (grant no. 81370868) and the Fundamental Research Funds for the Central Universities and Jiangsu Province Scientific Research Innovation Project for Graduate Students (grant no. KYLX_0197).

\section{References}

1. Levey AS, Atkins R, Coresh J, Cohen EP, Collins AJ, Eckardt KU, Nahas ME, Jaber BL, Jadoul M, Levin A, et al: Chronic kidney disease as a global public health problem: Approaches and initiatives-a position statement from kidney disease improving global outcomes. Kidney Int 72: 247-259, 2007.

2. Schieppati A and Remuzzi G: Chronic renal diseases as a public health problem: Epidemiology, social and economic implications. Kidney Int Suppl 98: S7-S10, 2005.

3. Jacobson HR: Chronic renal failure: Pathophysiology. Lancet 338: 419-423, 1991.

4. Luo X, Deng L, Lamsal LP, Xu W, Xiang C and Cheng L: AMP-activated protein kinase alleviates extracellular matrix accumulation in high glucose-induced renal fibroblasts through mTOR signaling pathway. Cell Physiol Biochem 35: 191-200, 2015.

5. Zhou X, Zhang J, Xu C and Wang W: Curcumin ameliorates renal fibrosis by inhibiting local fibroblast proliferation and extracellular matrix deposition. J Pharmacol Sci 126: 344-350, 2014.

6. Eddy AA: Molecular insights into renal interstitial fibrosis. J Am Soc Nephrol 7: 2495-2508, 1996.

7. Ronco P and Chatziantoniou C: Matrix metalloproteinases and matrix receptors in progression and reversal of kidney disease: Therapeutic perspectives. Kidney Int 74: 873-878, 2008.

8. Basile DP, Fredrich K, Weihrauch D, Hattan N and Chilian WM: Angiostatin and matrix metalloprotease expression following ischemic acute renal failure. Am J Physiol Renal Physiol 286: F893-F902, 2004.

9. Racca MA, Novoa PA, Rodríguez I, Della Vedova AB, Pellizas CG, Demarchi M and Donadio AC: Renal dysfunction and intragraft proMMP9 activity in renal transplant recipients with interstitial fibrosis and tubular atrophy. Transpl Int 28: 71-78, 2015.

10. Zhou TB, Qin YH, Lei FY, Huang WF and Drummen GP: Prohibitin attenuates oxidative stress and extracellular matrix accumulation in renal interstitial fibrosis disease. PLoS One 8: e77187, 2013.

11. DeCoux A, Lindsey ML, Villarreal F, Garcia RA and Schulz R Myocardial matrix metalloproteinase-2: Inside out and upside down. J Mol Cell Cardiol 77: 64-72, 2014.
12. Fine LG and Norman JT: Chronic hypoxia as a mechanism of progression of chronic kidney diseases: From hypothesis to novel therapeutics. Kidney Int 74: 867-872, 2008.

13. Norman JT, Orphanides C, Garcia P and Fine LG: Hypoxiainduced changes in extracellular matrix metabolism in renal cells. Exp Nephrol 7: 463-469, 1999.

14. Orphanides C, Fine LG and Norman JT: Hypoxia stimulates proximal tubular cell matrix production via a TGF-betal-independent mechanism. Kidney Int 52: 637-647, 1997.

15. Li J, Fan R, Zhao S, Liu L, Guo S, Wu N, Zhang W and Chen P: Reactive oxygen species released from hypoxic hepatocytes regulates MMP-2 expression in hepatic stellate cells. Int $\mathrm{J}$ Mol Sci 12: 2434-2447, 2011.

16. Bellot G, Garcia-Medina R, Gounon P, Chiche J, Roux D, Pouysségur J and Mazure NM: Hypoxia-induced autophagy is mediated through hypoxia-inducible factor induction of BNIP3 and BNIP3L via their BH3 domains. Mol Cell Biol 29: 2570-2581, 2009.

17. Mazure NM and Pouysségur J: Hypoxia-induced autophagy: Cell death or cell survival? Curr Opin Cell Biol 22: 177-180, 2010.

18. Michiels C: Physiological and pathological responses to hypoxia. Am J Pathol 164: 1875-1882, 2004.

19. Dada LA, Welch LC, Zhou G, Ben-Saadon R, Ciechanover A and Sznajder JI: Phosphorylation and ubiquitination are necessary for Na, K-ATPase endocytosis during hypoxia. Cell Signal 19: 1893-1898, 2007.

20. Kukulski W, Schorb M, Kaksonen M and Briggs JA: Plasma membrane reshaping during endocytosis is revealed by time-resolved electron tomography. Cell 150: 508-520, 2012.

21. Kim HN and Chung HS: Caveolin- 1 inhibits membrane-type 1 matrix metalloproteinase activity. BMB Rep 41: 858-862, 2008.

22. Sato H, Takino T, Okada Y, Cao J, Shinagawa A, Yamamoto E and Seiki M: Matrix metalloproteinase expressed on the surface of invasive tumour cells. Nature 370: 61-65 1994.

23. Butler GS, Butler MJ, Atkinson SJ, Will H, Tamura T, Schade van Westrum S, Crabbe T, Clements J, d'Ortho MP and Murphy G: The TIMP2 membrane type 1 metalloproteinase 'receptor' regulates the concentration and efficient activation of progelatinase A.A kinetic study. J Biol Chem 273: 871-880, 1998.

24. D'Amico G: Tubulo-interstitial damage in glomerular diseases: Its role in the progression of the renal damage. Nephrol Dial Transplant 13 (Suppl 1): S80-S85, 1998.

25. Livak KJ and Schmittgen TD: Analysis of relative gene expression data using real-time quantitative PCR and the 2(-Delta Delta C(T)) Method. Methods 25: 402-408, 2001.

26. Takahara T, Furui K, Funaki J, Nakayama Y, Itoh H, Miyabayashi C, Sato H, Seiki M, Ooshima A and Watanabe A: Increased expression of matrix metalloproteinase-II in experimental liver fibrosis in rats. Hepatology 21: 787-795, 1995.

27. Tanaka S, Tanaka T and Nangaku M: Hypoxia as a key player in the AKI-to-CKD transition. Am J Physiol Renal Physiol 307: F1187-F1195, 2014.

28. Norman J and Orphanides C: Hypoxia alters extracellular matrix (ECM) synthesis and turnover in renal fibroblasts. J Am Soc Nephrol 7: A2577, 1996.

29. Chow AK, Cena J, El-Yazbi AF, Crawford BD, Holt A, Cho WJ, Daniel EE and Schulz R: Caveolin-1 inhibits matrix metalloproteinase-2 activity in the heart. J Mol Cell Cardiol 42: 896-901, 2007.

30. Schnitzer JE, Oh P, Pinney E and Allard J: Filipin-sensitive caveolae-mediated transport in endothelium: Reduced transcytosis, scavenger endocytosis, and capillary permeability of select macromolecules. J Cell Biol 127: 1217-1232, 1994. 